- Technology and Methods $•$

\title{
Measurement and analysis of the imaging dose with megavoltage computed tomography for helical tomotherapy
}

\author{
Shou-Ping Xu, ${ }^{1}$ Chuan-Bin Xie, ${ }^{1}$ Zhong-Jian Ju, ${ }^{1}$ Xiang-Kun Dai, ${ }^{1}$
}

Han-Shun Gong, ${ }^{1}$ Yan-Yan Guo ${ }^{2}$ and Lian-Yuan Wang ${ }^{1}$

1. Department of Radiation Oncology,

PLA General Hospital,

Beijing, 100853,

P.R. China

2. Department of Radiation Oncology,

The Second Hospital of

Tianjin Medical University,

Tianjin, 300211,

P.R. China

Correspondence to: Shou-Ping Xu

Tel. : 86.10.66937762

Fax. : 86.10.68211455

Email:xshp228@163.com

This paper was translated into English from its original publication in Chinese.

Translated by: Zhen-Hua Pan

and Hua He on 2009-08-03

The original Chinese version of this paper is published in:Ai Zheng (Chinese Journal of Cancer) 28(8); http://www.cjcsysu.cn/cn/article. asp? id=15493)

Submitted : 2008-09-25

Revised : 2009-04-10
[Abstract] Background \& Objective: During the helical tomotherapy process, megavoltage computed tomography (MVCT) images are usually used for guiding the precise setup of patients before/after treatment delivery, which would certainly increase the total dose for patients. This study was to investigate the imaging dose of MVCT using the body and head phantom on a tomotherapy machine. Methods: A set of cylindrical body and head phantoms was adopted for scanning with different pitch values (1.0/2.0/ $3.0)$, lengths $(4.8 / 7.2 / 9.6 / 12 / 14.4 \mathrm{~cm})$ and patient setups on the couch of tomotherapy system. The average MVCT imaging doses were measured using A1SL chambers inserted in the phantoms with preset geometry. The dose uniformity was qualitatively analyzed. Results: The MVCT scanning dose for the body phantom was between 0.599 and 2.876 cGy during each treatment delivery, while the dose for the head phantom was between 0.913 and 3.231 cGy. Two major parameters, the assigned pitch numbers and scanning lengths, were the most important impacts to the dose variation. The MVCT dose was inversely proportional to the CT pitch value. With respect to the scanning length, the doses responded differently along the radial direction of the phantoms with different setup criteria. Conclusion: The results may provide a reliable guidance for proper planning design of the scanning region, which is valuable to help minimize the extra doses to patient. Key words: helical tomotherapy, radiotherapy, MVCT, imaging dose

As optimal dose distributions and very sharp dose gradients can be now attained by recently developed systems such as intensity-modulated radiation therapy (IMRT), the requirement for precise individual setup has given rise to the development of image-guided radiation therapy (IGRT). Therefore, improving the accuracy becomes the inevitable trend of this technique.

A helical tomography (HT) system Hi-Art II, the adapted integration of modern helical computed tomography and medical linear accelerator, has been one of the important innovations to deliver IMRT with image guidance. The Hi-Art system, applied for clinical practice in our hospital since September 2007, was the first one in China, from which helical megavoltage computed tomography (MVCT) images can be acquired by the same radiation 
source as well as intensity-modulated beams. To inspect and verify the patient alignment before and during the entire treatment, continuous 360 degree beam images around the transverse section of patients are demanded. It is well-known that resolution for volume imagery must be high enough to get soft tissue information. Compton scattering effect is mainly responsible for explaining the action of photons in soft tissues within the energy range from kilovoltage $(\mathrm{kV})$ to megavoltage (MV). According to it, larger doses have to be used in MVCT to achieve equal signal-to-noise ratios (SNR) and resolutions for MVCT images than kVCT ones. In this case, serious considerations have to be made to optimally balance the quality of image and additional doses of daily radiation. In this study, we carried out a series of clinical trials by combining a typical set of body and head phantom and an A1SL ion chamber to investigate practical imaging doses received by patients during the MVCT process and tried to figure out the certain factors playing main roles to influence radiation doses.

\section{Materials and Methods}

MVCT imaging process. As with the helical CT imaging process, MVCT scanning was implemented by manipulating simultaneous movements of patient table and gantry. During the MVCT scanning, energy of electronic beam current decreased from $6 \mathrm{MeV}$ to $3.5 \mathrm{MeV}$. Longitudinal direction length of the primary collimator was $4 \mathrm{~mm}$, and the rotation period of gantry was 10 seconds. ${ }^{1}$ All the leaves of multi leaf collimators (MLC) were kept opening during imaging. Three pitch values of 1.0, 2.0, and 3.0 presented to obtain the table speed of 4,8 , and $12 \mathrm{~mm}$ per circle. MVCT images were collected at the reconstruction depth of $2 \mathrm{~mm}$ (Fine), 4 $\mathrm{mm}$ (Normal), and $6 \mathrm{~mm}$ (Coarse) respectively. More details about helical CT systems and MVCT imaging process have been elucidated before. $^{1-3}$ The parameters during MVCT scanning were pitch values and scanning range.

Measuring apparatus. A set of body and head phantom was adopted to simulate a patient during the period of MVCT scanning. The head was a self-made synthetic glass cylinder with the diameter of $15 \mathrm{~cm}$ and the length of $14 \mathrm{~cm}$. The cylinder body phantom (Med Cal, USA) was a solid water slab made of two hemispheres. The diameter and length was $30 \mathrm{~cm}$ and $18 \mathrm{~cm}$, respectively. A row of ion chamber plugs were located along the radial directions of the plane vertical to that where diameters of the body phantom (Fig. 1a). Five centimeter from the phantom center on the plane vertical to the CT scanning one also resided rotationally ion chamber plugs with a jaw width of $1 \mathrm{~cm}$, length of which is kept $0.5 \mathrm{~cm}$ from the center, $7.5 \mathrm{~cm}$ and $9.5 \mathrm{~cm}$ i.e., to assure the sensitive part of ion chamber rightly covers the phantom center, and an equivalent solid water stick is inserted in each of them. TomoElectrometer (Standard Imaging, USA), an 8 channel dosimeter, 3 sets of A1SL ion chamber with the volume of $0.056 \mathrm{~cm}^{3}$, of which length of the sensitive part is $4.1 \mathrm{~mm}$, and the outer diameter is $6.25 \mathrm{~mm}$, were used for measurement.

Experimental criteria. Different pitch values (1.0/2.0/3.0), scanning lengths $(4.8 / 7.2 / 9.6 / 12 / 14.4 \mathrm{~cm})$, and patient setups on the table were used to accomplish MVCT scanning of the phantom in order to measure radiation doses with each assigned criteria. To choose a proper patient setup, location of the tumor in the cross sectional images and thickness of the body were referred to, and their variations led to variant vertical positions of the patient table during CT scanning. Here we presented three positions of the phantom center, one of which was aligned to the beam central axis, while the other two were $5 \mathrm{~cm}$ upper and $5 \mathrm{~cm}$ lower respectively. Doses at twenty-eight sets of points of the body phantom were measured. These points were distributed with an arc width of 45 degree along the radial direction, and with a linear distance of $4 \mathrm{~cm}$ along the axis direction (Fig 1 a). Unlike the body phantom, doses at only 4 points which are $4 \mathrm{~cm}$ vertically away from the head phantom center besides the center itself were measured according to clinical facts (Fig 1 b). Qualitative assessments were carried out by analysing points with the smallest, the 

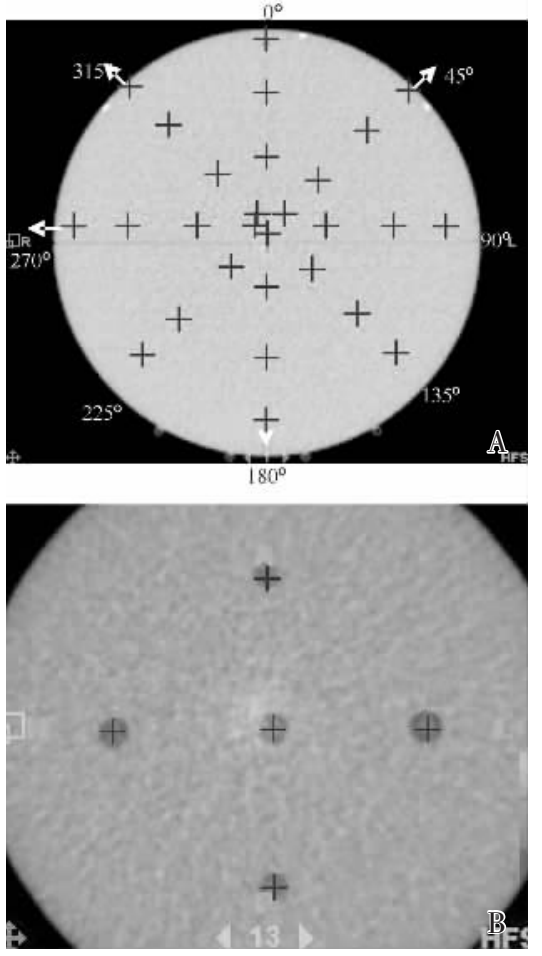

Figure 1 Illustrations of the measured points in the phantoms A : a cylindrical body phantom; B : a head phantom

largest and the average image dose, as well as dose uniformities with different patient setups.

\section{Results}

MVCT imaging dose. The ion chamber was set inside the phantom, by which the multiple scan average dose (MSAD) of the phantom was measured under each scanning criteria. Once the whole phantom was scanned, the dose ion chamber would show the average of all points, which contains not only the imaging dose of sensitive part of the ion chamber, but also the scattering doses of all the other contiguous planes. ${ }^{1,46}$

Measurement of body phantom. Doses of the 28 sets of points in the body phantom under each scanning condition, which were measured by ion chamber, were shown in Table 1. Of all the doses, the smallest one was 0.599cGy, while the largest one was $2.876 \mathrm{cGy}$, and their average was $1.935 \mathrm{cGy}$.

Measurement of head phantom. Doses of the 5 points in the head phantom under each scanning condition were measured by ion chamber were shown in Table 2 . The doses ranged from $0.895 \mathrm{cGy}$ to $3.231 \mathrm{cGy}$.

Result of the measurement indicates that value of the scanning dose is inversely proportional to that of the pitch. Hence, for the same scanning length, patient who received MVCT scanning with the pitch value of 1.0 would actually get 2 times the radiation dose of the one with the pitch value of 2.0, and 3 times the radiation dose of the one with pitch value of 3.0 .

Analysis of dose uniformity under different setups. As shown in Fig. 1 (a), where the direction indicated by the arrow is determined forward, the cylindrical body phantom was used to measure doses of points distributed separately $14 \mathrm{~cm}, 10 \mathrm{~cm}, 5 \mathrm{~cm}, 0.5$ cm. $-4.5 \mathrm{~cm},-9.5 \mathrm{~cm}$, and $-13.5 \mathrm{~cm}$ away from the axis with the pitch value of 2.0 and scanning the length of $7.2 \mathrm{~cm}$. Dose uniformity was analyzed by the observation of dose variations both along the same radial directions and different ones with different center setups (Fig 2).

Our study also revealed a corresponding change of the MVCT average doses with that of the distances along the radial directions attributed to the influence of radiation attenuation: the scanning dose delivered laterally (270) decreased as the distance increased with setups of table-arise and table-down, while those delivered with the other setups normally appeared an ascending trend. Fig. 2 shows the result that dose distribution at central setup performed more symmetrical than deviated setups.

\section{Discussion}

IGRT has greatly improved the accuracy of target positioning during radiation therapy. Clinical trails indicate that the IGRT technique could reduce not only systematic errors but also random deviations by the application of daily images. The demanded accuracy depends on the expanding possibility of the target and the distance from the target to sensitive organs; it is obviously necessary to carry out image captures daily when the sensitive organs are quite close.,7

Though image guiding is now believed to be 
Table 1 MVCT dose measured in the cylindrical body phantom

\begin{tabular}{|c|c|c|c|c|c|}
\hline \multirow{2}{*}{ Alignment } & \multirow{2}{*}{ Pitch } & \multirow{2}{*}{ Length $(\mathrm{cm})$} & \multicolumn{3}{|c|}{ Measured dose (cGy) } \\
\hline & & & Minimum dose & Maximum dose & Mean dose \\
\hline \multirow[t]{15}{*}{ Center setup } & 1.0 & 4.8 & 1.985 & 2.585 & 2.239 \\
\hline & 1.0 & 7.2 & 2.157 & 2.652 & 2.367 \\
\hline & 1.0 & 9.6 & 2.325 & 2.743 & 2.466 \\
\hline & 1.0 & 12 & 2.402 & 2.818 & 2.541 \\
\hline & 1.0 & 14.4 & 2.475 & 2.876 & 2.600 \\
\hline & 2.0 & 4.8 & 1.007 & 1.318 & 1.144 \\
\hline & 2.0 & 7.2 & 1.089 & 1.383 & 1.194 \\
\hline & 2.0 & 9.6 & 1.147 & 1.433 & 1.273 \\
\hline & 2.0 & 12 & 1.195 & 1.469 & 1.319 \\
\hline & 2.0 & 14.4 & 1.213 & 1.483 & 1.333 \\
\hline & 3.0 & 4.8 & 0.678 & 1.047 & 0.767 \\
\hline & 3.0 & 7.2 & 0.691 & 1.069 & 0.784 \\
\hline & 3.0 & 9.6 & 0.704 & 1.089 & 0.818 \\
\hline & 3.0 & 12 & 0.709 & 1.105 & 0.840 \\
\hline & 3.0 & 14.4 & 0.718 & 1.111 & 0.847 \\
\hline \multirow[t]{15}{*}{ Up $5 \mathrm{~cm}$ setup } & 1.0 & 4.8 & 1.882 & 2.554 & 2.187 \\
\hline & 1.0 & 7.2 & 1.979 & 2.621 & 2.279 \\
\hline & 1.0 & 9.6 & 2.104 & 2.687 & 2.367 \\
\hline & 1.0 & 12 & 2.118 & 2.734 & 2.436 \\
\hline & 1.0 & 14.4 & 2.125 & 2.749 & 2.462 \\
\hline & 2.0 & 4.8 & 0.948 & 1.282 & 1.089 \\
\hline & 2.0 & 7.2 & 1.010 & 1.318 & 1.137 \\
\hline & 2.0 & 9.6 & 1.052 & 1.362 & 1.186 \\
\hline & 2.0 & 12 & 1.078 & 1.397 & 1.221 \\
\hline & 2.0 & 14.4 & 1.083 & 1.423 & 1.238 \\
\hline & 3.0 & 4.8 & 0.599 & 0.922 & 0.720 \\
\hline & 3.0 & 7.2 & 0.612 & 0.964 & 0.737 \\
\hline & 3.0 & 9.6 & 0.649 & 0.961 & 0.783 \\
\hline & 3.0 & 12 & 0.667 & 0.989 & 0.806 \\
\hline & 3.0 & 14.4 & 0.668 & 0.109 & 0.817 \\
\hline \multirow[t]{15}{*}{ Down $5 \mathrm{~cm}$ setup } & 1.0 & 4.8 & 1.865 & 2.775 & 2.183 \\
\hline & 1.0 & 7.2 & 2.060 & 2.867 & 2.321 \\
\hline & 1.0 & 9.6 & 2.169 & 2.725 & 2.388 \\
\hline & 1.0 & 12 & 2.241 & 2.767 & 2.431 \\
\hline & 1.0 & 14.4 & 2.244 & 2.795 & 2.468 \\
\hline & 2.0 & 4.8 & 0.980 & 1.370 & 1.130 \\
\hline & 2.0 & 7.2 & 1.044 & 1.493 & 1.168 \\
\hline & 2.0 & 9.6 & 1.088 & 1.428 & 1.213 \\
\hline & 2.0 & 12 & 1.139 & 1.446 & 1.257 \\
\hline & 2.0 & 14.4 & 1.169 & 1.457 & 1.276 \\
\hline & 3.0 & 4.8 & 0.645 & 0.974 & 0.767 \\
\hline & 3.0 & 7.2 & 0.688 & 1.008 & 0.807 \\
\hline & 3.0 & 9.6 & 0.694 & 1.024 & 0.831 \\
\hline & 3.0 & 12 & 0.703 & 1.029 & 0.853 \\
\hline & 3.0 & 14.4 & 0.706 & 1.037 & 0.860 \\
\hline
\end{tabular}

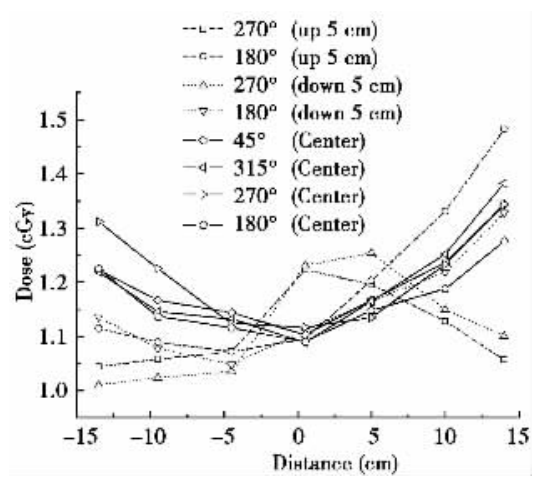

Figure 2 Analysis of the point doses crossing through the cylindrical phantom with different setups

a principal part of precise radiation therapy and systems have been designed frequently for in-room purposes, the probability of increased daily doses have to arouse more attention. Our study showed a daily dose range between 0.5-3.3 cGy was obtained by the body phantome with different setups of MVCT, in which case a patient would receive a total dose of 10-120 cGy if the regular radiation is repeated 20-35 times, and the result fits quite well to those of other reports. ${ }^{5,8}$ In general, doses of MVCT under normal scanning conditions stay at the level of 1 percent of the prescription. Our study suggests that the radiation doses of a patient is closely related to the pitch values and the scanning length, and the smaller pitch value and the larger scanning field increases the doses. Analysis of data from table 1 and 2 reveals that while with the same scanning criteria of MVCT, a fatter patient would receive less radiation doses than a thinner one. For different parts of the same 
Table 2 MVCT dose measured in the head phantom

\begin{tabular}{|c|c|c|c|c|c|}
\hline \multirow{2}{*}{ Alignment } & \multirow{2}{*}{ Pitch } & \multirow{2}{*}{ Length $(\mathrm{cm})$} & \multicolumn{3}{|c|}{ Measured dose (cGy) } \\
\hline & & & Minimum dose & Maximum dose & Mean dose \\
\hline \multirow[t]{9}{*}{ Center setup } & 1.0 & 4.8 & 2.839 & 2.975 & 2.895 \\
\hline & 1.0 & 7.2 & 2.999 & 3.097 & 3.052 \\
\hline & 1.0 & 9.6 & 3.097 & 3.177 & 3.135 \\
\hline & 2.0 & 4.8 & 1.451 & 1.498 & 1.473 \\
\hline & 2.0 & 7.2 & 1.494 & 1.573 & 1.524 \\
\hline & 2.0 & 9.6 & 1.537 & 1.597 & 1.567 \\
\hline & 3.0 & 4.8 & 0.905 & 1.043 & 0.961 \\
\hline & 3.0 & 7.2 & 0.928 & 1.072 & 0.994 \\
\hline & 3.0 & 9.6 & 0.956 & 1.172 & 1.053 \\
\hline \multirow[t]{9}{*}{ Up $5 \mathrm{~cm}$ setup } & 1.0 & 4.8 & 2.817 & 2.945 & 2.869 \\
\hline & 1.0 & 7.2 & 2.928 & 3.054 & 2.985 \\
\hline & 1.0 & 9.6 & 2.787 & 3.098 & 3.017 \\
\hline & 2.0 & 4.8 & 1.424 & 1.486 & 1.453 \\
\hline & 2.0 & 7.2 & 1.495 & 1.569 & 1.513 \\
\hline & 2.0 & 9.6 & 1.519 & 1.600 & 1.558 \\
\hline & 3.0 & 4.8 & 0.895 & 1.047 & 0.958 \\
\hline & 3.0 & 7.2 & 0.938 & 1.078 & 0.994 \\
\hline & 3.0 & 9.6 & 0.942 & 1.094 & 1.010 \\
\hline \multirow[t]{9}{*}{ Down $5 \mathrm{~cm}$ setup } & 1.0 & 4.8 & 2.795 & 3.036 & 2.917 \\
\hline & 1.0 & 7.2 & 2.918 & 3.137 & 3.016 \\
\hline & 1.0 & 9.6 & 3.002 & 3.231 & 3.092 \\
\hline & 2.0 & 4.8 & 1.371 & 1.530 & 1.449 \\
\hline & 2.0 & 7.2 & 1.495 & 1.570 & 1.529 \\
\hline & 2.0 & 9.6 & 1.466 & 1.608 & 1.539 \\
\hline & 3.0 & 4.8 & 0.852 & 1.041 & 0.949 \\
\hline & 3.0 & 7.2 & 0.877 & 1.070 & 0.977 \\
\hline & 3.0 & 9.6 & 0.893 & 1.084 & 0.993 \\
\hline
\end{tabular}

patient, it is the chest and abdomen that would receive the smallest radiation doses, following the head, and normally neck would receive the largest dose. However we would not give any words on whether an amended plan is needed to take consider of increased daily radiation doses by MVCT in that there have not been any further research on possible risks of daily radiations from MVCT. We are sure that along with the advancement of self-adapting radiation therapy, MVCT dosimetry and even the biological effect of this technology, the problem of how to practically reduce the radiation of normal tissues during image-guided helical CT would be better interpreted.

After all, our study of investigating radiation doses achieved from MVCT by a set of body and head phantom provides reliable clinical evidences for proper planning design of the scanning region, especially the radiation-sensitive organs such as lens, and also helps reduce extra doses to patients.

\section{References}

[1] Meeks SL, Harmon JJ, Langen KM, et al. Performance characterization of megavoltage computed tomography imaging on a helical tomotherapy unit [J]. Med Phys, 2005,32 (8):2673 2681.

[2] Mackie TR, Balog J, Ruchala K, et al. Tomotherapy $[\mathrm{J}]$. Semin Radiat Oncol, 1999,9(1) : 108-117.

[3] Ruchala KJ, Olivera GH, Schloesser EA, et al. Megavoltage CT on a tomotherapy system $[\mathrm{J}]$. Phys Med Biol, 1999,44(10):2597-2621.

[4] Mutic S, Palta JR, Butker EK, et al. Quality assurance for computedtomography simulators and the computetomography-simulation process: report of the AAPM Radiation Therapy Committee Task Group No.66 [J]. Med Phys, 2003,30(10):2762-2792.

[5] Shah AP, Langen KM, Ruchala KJ, et al. Patient dose from megavoltage computed tomography imaging $[\mathrm{J}]$. Int. J. Radiat Oncol Biol Phys, 2008,70 (5) : 1579-1587.

[6] Liu B, Bai M, Fei XL, et al. Optimization of patient dose parameters of CT examination [J]. Zhong Guo Yi Liao Zhuang Bei, 2008, 23(3): 54-56. [in Chinese]

[7] Zeidan OA, Langen KM, Meeks SL, et al. Evaluation of image-guidance protocols in the treatment of head and neck cancers $[\mathrm{J}]$. Int $\mathrm{J}$ Radiat Oncol Biol Phys, 2007,67(3):670-677.

[8] Beavis AW. Is tomotherapy the future of IMRT [J]? Br J Radio, 2004, $77(916)$ : 285-295. 